\title{
Plasma-enhanced atomic layer deposition for plasmonic TiN (Erratum)
}

Lauren Otto, Aeron Hammack, Shaul Aloni, D. Frank Ogletree, Deirdre Olynick, et al.

Lauren M. Otto, Aeron T. Hammack, Shaul Aloni, D. Frank Ogletree, Deirdre L. Olynick, Scott Dhuey, Bethanie J. H. Stadler, Adam M. Schwartzberg, "Plasma-enhanced atomic layer deposition for plasmonic TiN (Erratum)," Proc. SPIE 9919, Nanophotonic Materials XIII, 991918 (12 May 2020); doi: $10.1117 / 12.2573754$

Event: SPIE Nanoscience + Engineering, 2016, San Diego, California, United States 


\section{Plasma-enhanced atomic layer deposition for plasmonic TiN (Erratum)}

Lauren M. Otto, Univ. of Minnesota, Twin Cities (United States) and Lawrence Berkeley National Lab. (United States); Aeron T. Hammack, Shaul Aloni, D. Frank Ogletree, Deirdre L. Olynick, Scott Dhuey, Lawrence Berkeley National Lab. (United States); Bethanie J. H. Stadler, Univ. of Minnesota, Twin Cities (United States); Adam M. Schwartzberg, Lawrence Berkeley National Lab. (United States)

Proceedings Volume 9909, Nanophotonic Materials XIII; 99190N (2016) https://doi.org/10.1117/12.2573754

Online Publication Date: 3 October 2016

Erratum Published: 12 May 2020

A revised version of this manuscript was published on 12 May 2020. Details of the revision are as follows:

Change 1: Table 1, Plasma 2 - gases were listed in the wrong order and so were flipped.

Change 2: Equation 1 - an incorrect negative sign was switched to a positive sign in the denominator of the Lorentz component. 